\title{
Computer Networks E-Learning Based on Interactive Simulations and SCORM
}

\author{
doi:10.3991/ijoe.v7i2.1638 \\ P.Gil, F.A. Candelas and C.A. Jara \\ University of Alicante, Alicante, Spain
}

\begin{abstract}
This paper introduces a new set of compact interactive simulations developed for the constructive learning of computer networks concepts. These simulations, which compose a virtual laboratory implemented as portable Java applets, have been created by combining EJS (Easy Java Simulations) with the KivaNS API. Furthermore, in this work, the skills and motivation level acquired by the students are evaluated and measured when these simulations are combined with Moodle and SCORM (Sharable Content Object Reference Model) documents. This study has been developed to improve and stimulate the autonomous constructive learning in addition to provide timetable flexibility for a Computer Networks subject.
\end{abstract}

Index Terms-Computer networks, EJS, Java simulations, KivaNS, Learning assessment.

\section{INTRODUCTION}

The work presented in this paper is based on the pedagogical methodology used to adapt the subject of Computer Networks coursed in the Computer Science Engineering degree at the University of Alicante, in Spain, to the process of convergence to ESHE (European Space of Higher Education) [1]. In previous works, virtual laboratories, such as KivaNS [2] have been implemented and used in that subject. KivaNS is a free open source application with an user-friendly interface which has been programmed in Java. It was created in order to simulate computer networks interconnected with the TCP-IP architecture. This application has been developed as an autonomous virtual laboratory and permits the students of the courses about computer networks to carry out experiments about the IP routing, without the necessity of complex and expensive real physic equipment such as routers, switches, hubs, PCs, etc. Other free open-source software such as "ns" (network simulator) and its advantages and disadvantages compared to KivaNS are analyzed in [2].

In the two last years, since 2008 until nowadays, a blended learning methodology [3] has been applied as support to adapt the teaching and learning of the courses/subjects towards ECTS (European Credit Transfer System). For this reason, a LCMS (Learning Content Management System) as Moodle [4,5] has been used for learning of Computer Networks courses. This subject is structured in 45 hours of laboratory which are taught and evaluated with the support of Moodle. Furthermore, the subject has an average of 100 students per academic course. However, some problems can arise when KivaNS is used for teaching specific aspects. Firstly, students need to download and install the whole KivaNS application, and learn the use of its interface. Secondly, students have to use a complete interface oriented to design, draw, configure and simulate complete network structures. Thus, KivaNS can result an oversized application for using it to explore how specific protocols work. Moreover, the students have to use two applications, between of which there is not integration, and this issue may be considered other important disadvantage. These two independent applications are the SCORM (Sharable Content Object Reference Model) package including documents and quizexams inside the LCMS, and the KivaNS environment.

The work presented in this paper has two basic objectives. On the one hand, the design and creation of a more compact learning system which provides an integration of resources such as SCORM guides (based on HTML and Java) and simulations about Computer Networks topics (Java applets), being both resources embedded as interactive items. This way, students would not require spending time learning how to use new and complex applications. To reach this objective, authors have combined the simulation kernel of KivaNS, removing its graphical interface, with the powerful and friendly graphical interface of EJS (Easy Java Simulations) in order to create interactive simulations. EJS is a freeware, open-source tool specifically designed for the creation of interactive dynamic simulations [6], which has been designed for people who do not need advanced programming skills. On the other hand, this paper looks for measuring, by means of opinion surveys, the satisfaction degree of students when they use SCORM guides combined with the new simulations. In this last section, the paper will show comparison results and qualifications obtained by the students when they use SCORM with simulation applets and when they only use SCORM without them.

The paper is organized as follows: Section II explains how the interactive simulations have been designed by combining EJS with the KivaNS library. Section III describes the user interface of the Java applets developed for the Computer Network courses and other experiments are shown. Section IV comments how the subject is organized as a Moodle course. Section V shows the results obtained of a student learning study performed. Finally, some important conclusions are reported in Section VI. 


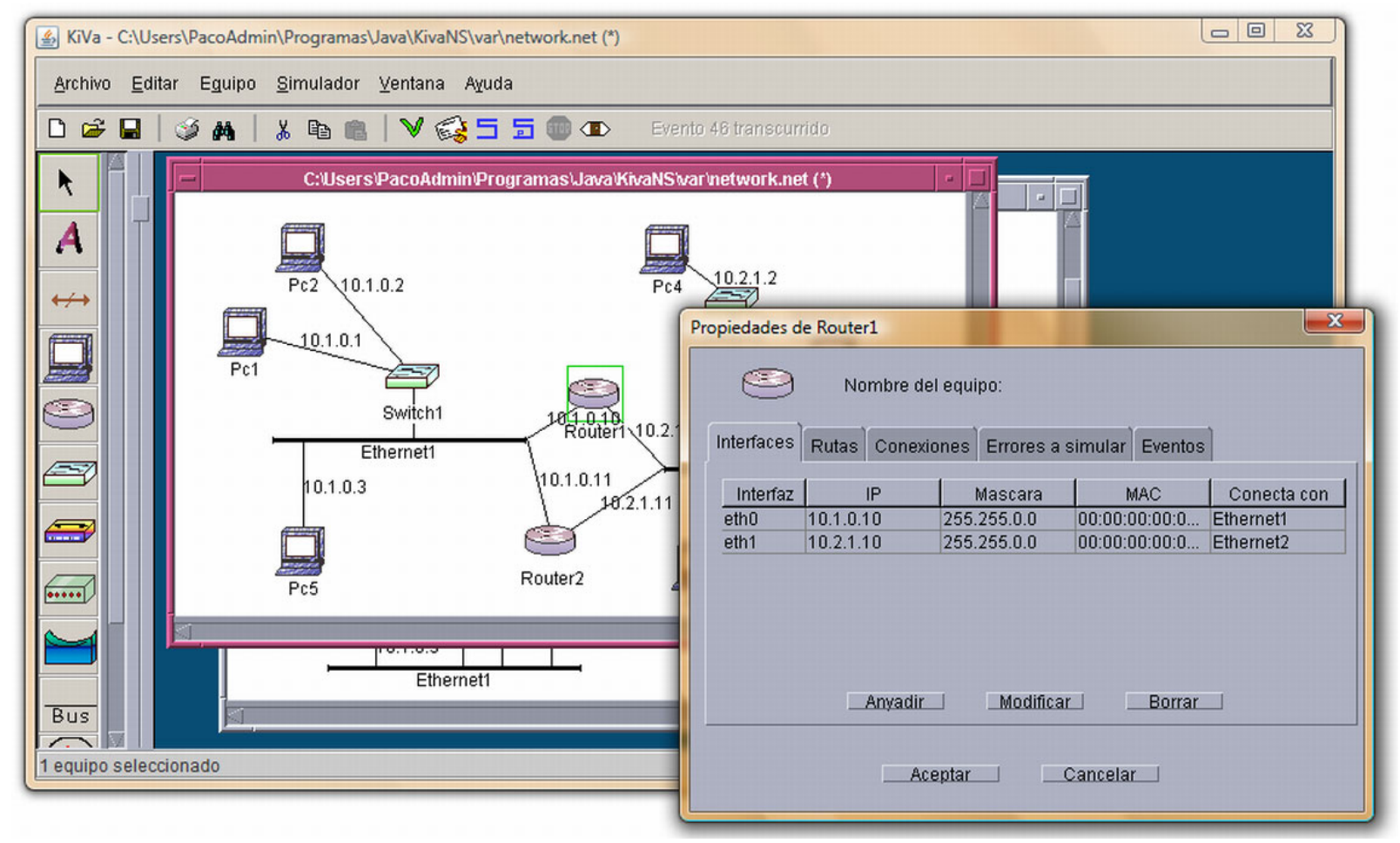

Figure 1. Graphical user interface of the KivaNS standalone application

\section{Design Of The Interative Network Simulations}

The developed virtual laboratories are focused on the design and simulation of data networks based on TCP/IP architecture and they are mainly composed of two software modules: a simulation engine specific for data networks driven by discrete events, and a graphical interactive user interface. The two modules are deployed as an application which has been programmed with the Java technology [7], now owned by Oracle. This feature provides a great portability, that is, the possibility of executing the application in a great number of platforms. The only requirement is to install the Java Runtime Environment (JRE) previously. The JRE can be freely downloaded from the Oracle web site.

For the simulation engine of the virtual labs presented in this paper, KivaNS has been used [2,8]. It is a software platform which allows users to create simulations and experiments without being present in the laboratory of real network devices (see next subsection). KivaNS has been designed as a stand-alone application, so that it cannot be included in a web page or in a LCMS, and it is not suitable to quickly teach specific simulations about working of networks. For this reason, authors have also employed EJS [9], which has been utilized to develop the interactive user interface of the applications and to generate "Java applets" from the network simulations. This way, the teaching units designed to explain how TCP/IP protocols works in LANs are compact interactive simulations, in the form of Java-applets, which are easily embedded in a LCMS like Moodle and are able to simulate real experimental cases, all without adding additional software for its installation, only the JRE plugin.

\section{A. KivaNS}

The main aims of KivaNS are to simulate how IP (Internet Protocol) works, and specially, to study the different techniques for routing the data packets through different network technologies. In order to do this, KivaNS also includes the simulation of auxiliary protocols such as ARP (Address Resolution Protocol), ICMP (Internet Control Message Protocol) in addition to IP, and emulates the basic working of link layer technologies such as PPP (Point to Point Protocol), Ethernet, or switched Ethernet.

The architecture of KivaNS is designed to have the simulation and the user interface services welldifferentiated. Thus, KivaNS is composed of two main blocks, both implemented in Java. The first block is an API (Application Programming Interface) that offers a simulation engine for data networks. The second block is a complete graphical user interface, which enables user to design data networks schemes, as well as to simulate and analyze them by using the API block in a transparent way.

The layout of the graphical user interface of the KivaNS application is shown in Fig. 1. This interface enables user to work with different network schemes in order to design, configure and simulate them. In the toolbar located on the left side, user can find the different devices available to draw the schemes. After placing one device into a scheme, the device can be configured with a set of parameters very similar to those that have been implemented for real devices. For example, the window placed on the right side in Fig. 1 shows the interface list of a routing device.

With regard to the API block, its main aspects will be described in the next point. 


\section{B. The KivaNS API}

As Fig. 2 shows, the API is composed of four main modules defined as follows: a discrete event manager, a group of objects to represent the networks, other objects to represent the equipment (host or interconnection equipment), and the communication layers stack. Thereby, the API is modular and extensible and new network or equipment technologies can be easily included. In fact, the API functionality has been increased in the last years. From the user viewpoint, API modules are presented as programming classes packaged in a Java JAR library, which can be used for any other Java application apart from KivaNS.

The modeling and simulation of the network scheme are structured in a very similar way to the architecture entities of real networks. Devices have objects of the different layers of protocols that they implements, and these objects can generate data packets to other layers, or receive packets from other layers. These received packets have to be processed according to the protocols specifications and their addressing/routing methods. At the lower layer, equivalent to the physic layer, there are network objects for routing of packets to objects in other devices. In addition, the objects called Network Manager and Equipment Manager administer the existent objects and facilitate the access to them. Fig. 3 shows an example of this working model.

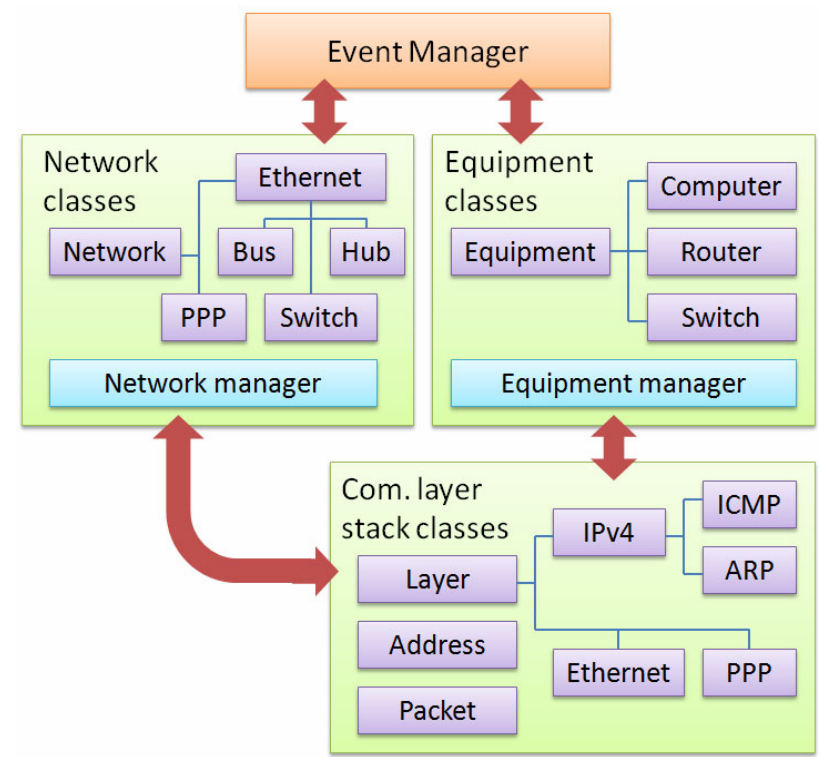

Figure 2. Structure of KivaNS simulation API

All interchanges of data among the objects are managed as discrete events, which are generated by the object outputs, and then queued. The event manager dispatches events to the objects inputs according a discrete time sequence, in an orderly way, step by step following the user interaction, or up to it reaches a state without more queued events.

The simulation output is the list of all the events generated and processed by the different objects. From this list, a trace of the packets of desired protocol can be obtained, in a similar way to the trace given by a protocol analyzer-sniffer in a real network. For each packet, the trace provides information about source, destination, data lengths, fragmentation, etc. All this information can be easily processed by the application that uses the API to produce the adequate visualization in the user interface. For example, a colored trace of the IPv4 packages can be displayed.

KivaNS also is able to simulate different kinds of errors in the networks and equipment, such as packets loss, and bad routing tables. Errors detected by protocols, such as timeouts or checksums, are also recorded in the simulation trace.

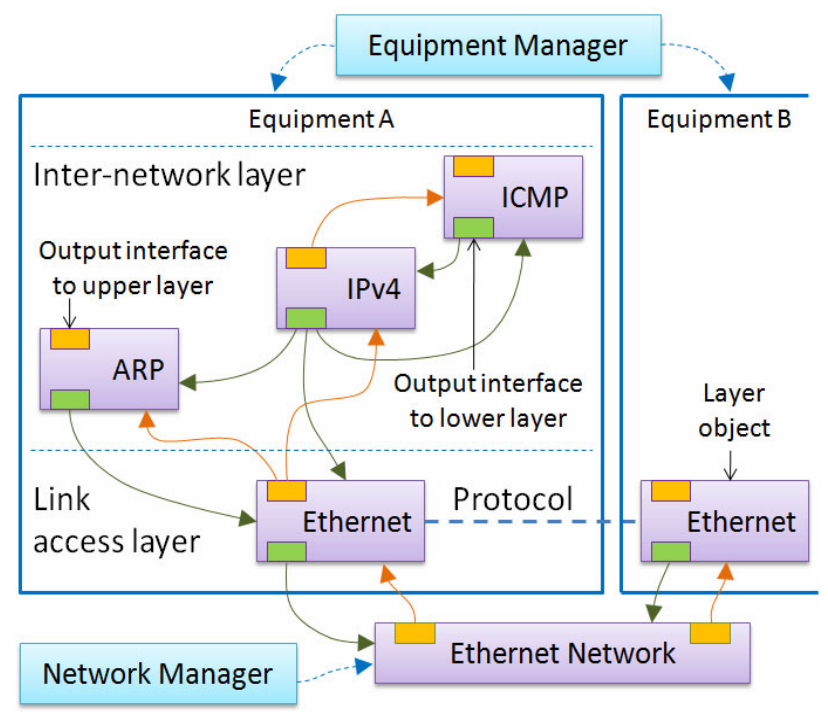

Figure 3. Architecture of a simulated network

\section{Simulation interface building and applet generation using Easy Java Simulations}

EJS is a freeware, open-source tool developed for the creation of interactive dynamic simulations. The interface of EJS allows users to develop simulations in an easy and quickly way. EJS is totally implemented in Java language, so that it provides a full portability of the applications generated. EJS can be freely downloaded and nowadays there are a lot of simulations developed with this software [9].

One of the most important features of EJS is the possibility to insert external libraries in Java as auxiliary frameworks to develop simulation code. This property has been used to integrate the existent simulation engine of KivaNS in the EJS environment (Fig. 4). The simulation engine is an independent module. This has been programmed as a JAR library for Java with the aim of providing a platform to develop other applications. Therefore, the KivaNS API can be used within EJS' environment to define the model of a network simulation. Thus, by combining the KivaNS simulation API with EJS, it is possible to quickly develop interactive graphical simulations which show topics of how the data networks works.

Another important feature of EJS is that it is a program which generates code. Once the user has developed a virtual laboratory, EJS automatically generates all the Java simulation compiled code, packs the resulting files in a compressed Java file and generates HTML pages containing embedded the virtual laboratory in a Java applet form. These applets are composed of a series of HTML pages and compressed Java files. Thus, the implementation and installation in Moodle can be easily 
performed. EJS users only need to upload these web pages and jar files (compiled Java code file) to the web server where Moodle is installed. Finally, this web page must be linked from the main page as an activity generated in a course for the student.

\begin{tabular}{|c|c|c|c|c|c|c|}
\hline \multicolumn{6}{|c|}{ Information about Router Simulation.xml } & \multirow[t]{2}{*}{ 区 } \\
\hline Metadata & Run options & \multicolumn{4}{|l|}{ Edit options } & \\
\hline \multicolumn{7}{|c|}{ Execution password } \\
\hline \multicolumn{7}{|c|}{$\nabla$ Add screen capture tools (VideoTool and EPS/GIF support) } \\
\hline \multicolumn{7}{|c|}{$\nabla$ Add data processing tools (DataTool and Fourier Tool) } \\
\hline \multicolumn{7}{|c|}{$\square$ Add languages facilities (TranslationTool and non-local languages) } \\
\hline \multicolumn{7}{|c|}{$\square$ Add support for eMersion (http://emersion.epfl.ch) } \\
\hline Imports: & :Redes." & & $\nabla$ & $\sqrt{ }$ & 睤 & $x$ \\
\hline \multirow{3}{*}{$\begin{array}{r}\text { JAR Libraries: } \\
\text { User files: } \\
\text { Files required: }\end{array}$} & KivaNS.jar & & $\nabla$ & $\sqrt{ }$ & 睠 & $\mathbf{x}$ \\
\hline & & & & $\nabla$ & $\sqrt{ }$ & $x$ \\
\hline & KivaNS.jar & & & & $\nabla$ & $\Rightarrow$ \\
\hline
\end{tabular}

Figure 4. Set-up of EJS' environment to include KivaNS library

\section{EJS APPLETS FOR NETWORK SIMULATION}

Fig. 5 and Fig. 6 show different appearances of the user interface of one of several Java applets developed with EJS and the KivaNS API. In particular, this example simulates how is made the data transmission between two hosts in a LAN Ethernet using IP by means of a router device. The students can send a data block and select the type of data, the block size and the source and destination host to simulate the transmission. Moreover, the student can change the MTU (Maximum Transfer Unit) value of each one of the network segments connected in the LAN Ethernet. This way, the data block is distributed as a set of packets in the simulation as IP fragmentation. Once configured the communication, the student can see, step by step, the transmission packets as a message sequence which represent the packets generated by ARP, ICMP and
IP. Thus, the traffic is shown in a very similar way to a protocol analyzer tool working in a real network.

All the traces generated are showed in the GUI of the applet, and the student can select a specific protocol by means of a filter option (see label "Protocol filter option" in Fig. 5). Moreover, the interface of the applet shows the generated communication traces as the data packets and the pathway. In Fig.5, it is shown the case when the IP filter is selected in the simulation and, in Fig. 6, it is shown the case of the selection of the ARP protocol.

Fig. 7 shows other network schemes which have been implemented in the interactive Java applets for the courses. As it can be seen in this figure, these configurations include several network devices such as a switch, a router and a hub at the same virtual laboratory. Specifically, the right scheme contains switch, hub and router devices and the left scheme switch and router components. This way, students can practice and experiment about the functionality of these devices when they work together at the same LAN. This feature has been implemented thanks to the KivaNS API because it contains several classes about network devices which can be instantiated within the same simulation in the EJS environment.

All the simulations developed with different schemes proposed for the course of Computer Networks allow student exploring cases such as the following:

- Operation of ARP.

- Functioning of bus or switched in Ethernet networks.

- Differences in the functionality among network devices.

- Working of IP, even considering fragmentation of data blocks.

- IP addressing and broadcasting.

- Routing of IP datagrams through one or more routers.

- Commonly used ICMP messages.

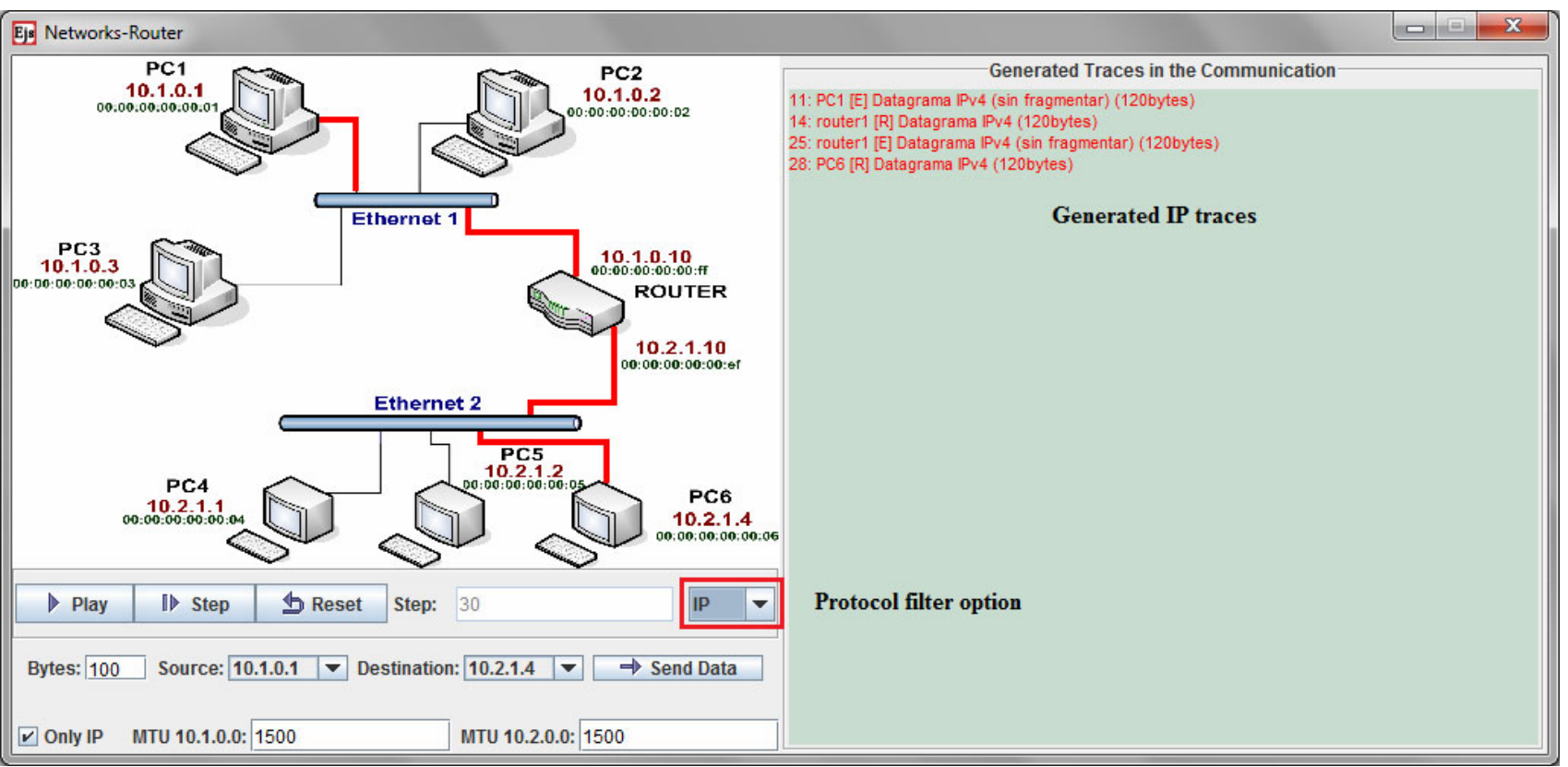

Figure 5. Appearance of the applet interface when the IP protocol filter is selected 


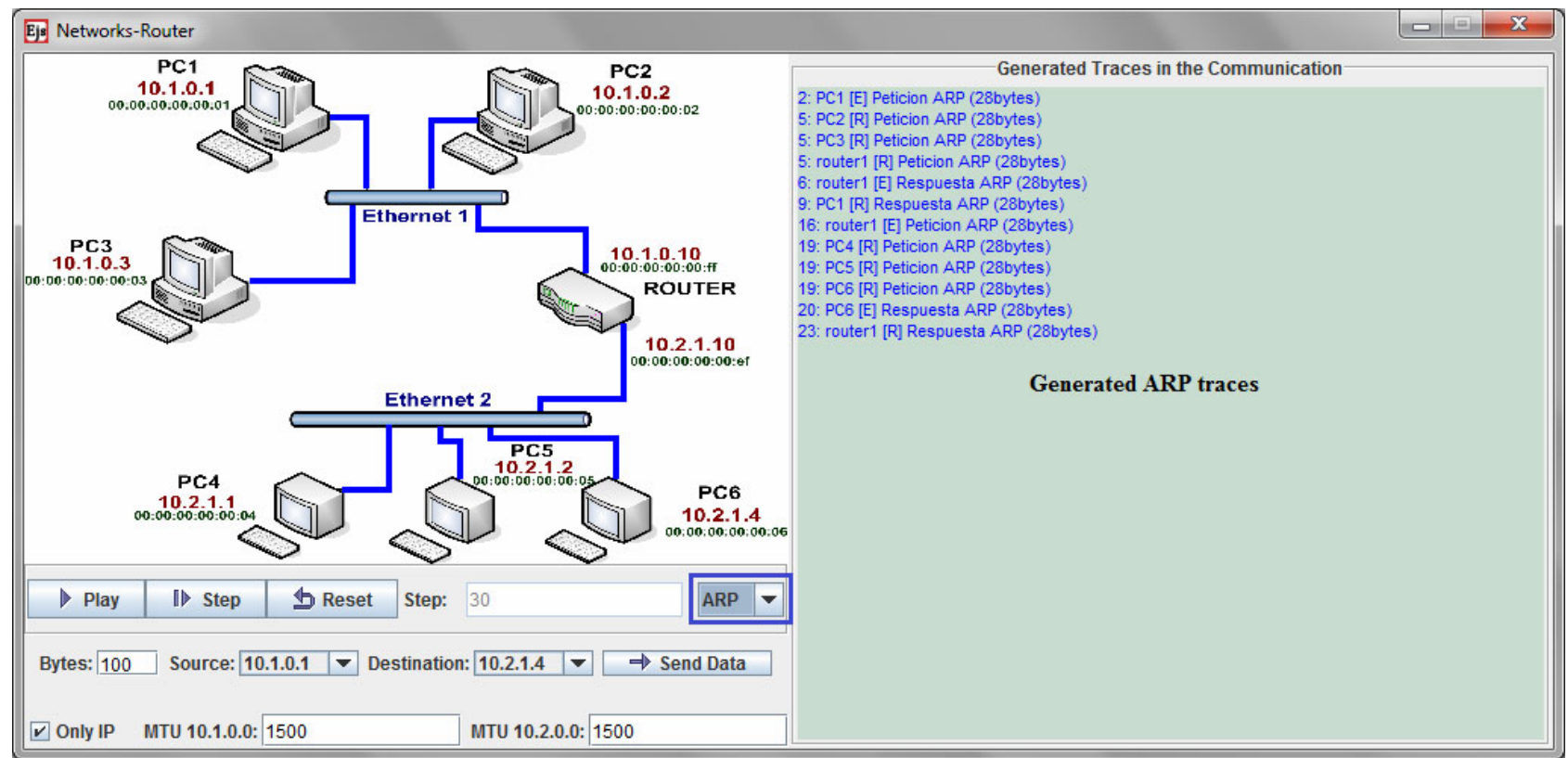

Figure 6. Appearance of the applet interface when the ARP protocol filter is selected
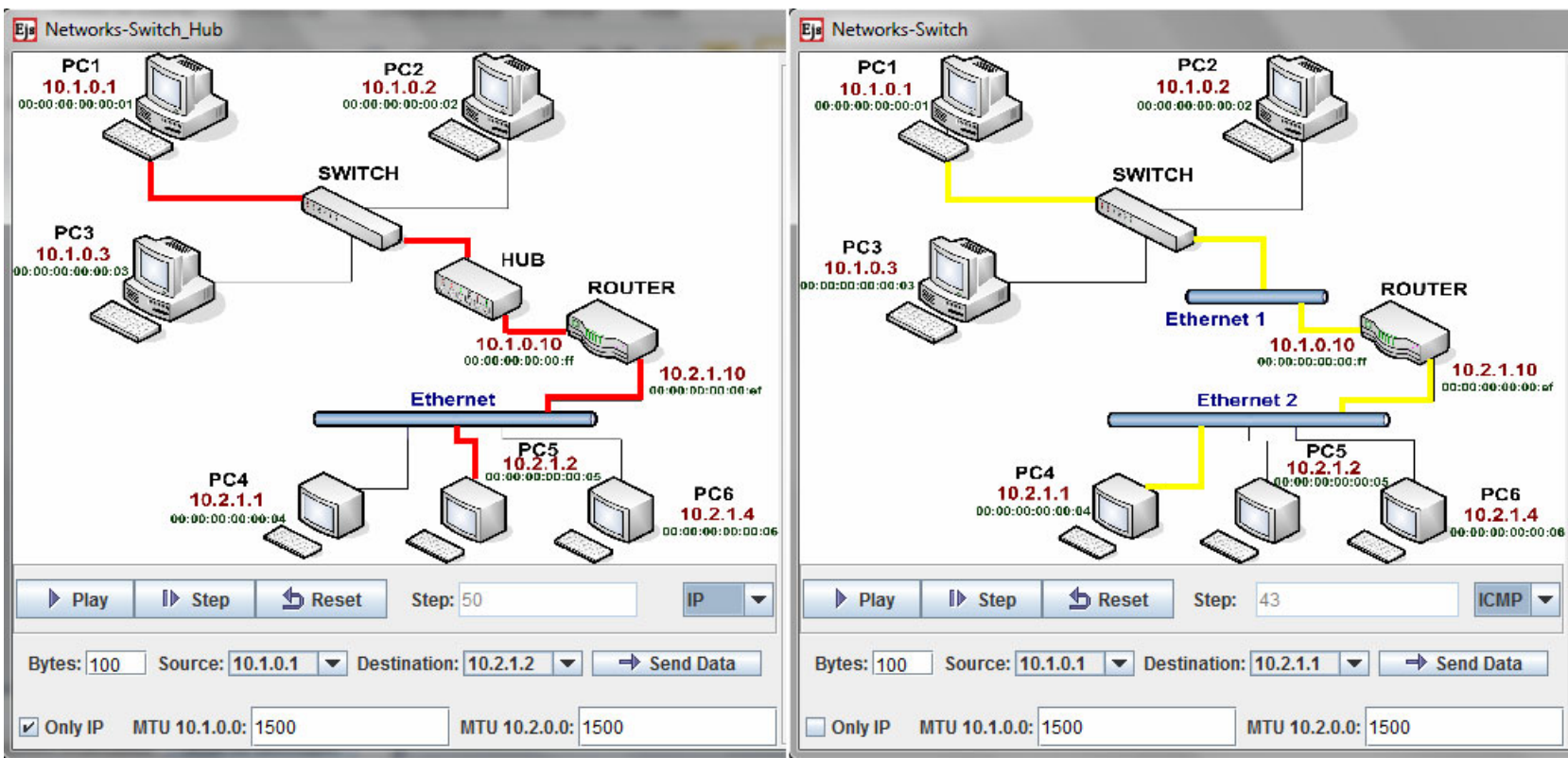

Figure 7. Other network schemes implemented in the Java applets

For all these reasons, the applets created can be a very useful tool from two viewpoints. First, the simulation allows teachers explaining how protocols work by means of graphical examples, while students can experiment with their own simulation applets, not only in practical sessions, but also in theory lessons. Second, the Java applets enables student to experiment without having a real network. Therefore, the students can be able to practice and experience outside the laboratory infrastructure of the faculty building. All information about the applets created with this approach can be accessed from [8]. In this web page there is a lot of information about how to use EJS and KivaNS engine and the readers can experiment with all simulations developed for Computer Network courses.
The access to the simulations by means of EJS applets is quite different from only using KivaNS application. Table I shows a comparison of the compliance degree of relevant features between the applets developed with EJS combined with the KivaNS API and the standalone KivaNS application. In general, the startup and learning of the simulation are much better if the applets are used. However, the standalone application allows user to design and to simulate more complex network schemes. In addition, applets can be integrated in web or LMS environments in an easy way. Thus, if the aim is to teach the basic principles of networks using courses inside a LMS environment, the interactive Java applets are the best choice. 
TABLE I.

COMPARISON BETWEEN THE EJS APPLETS AND KIVANS APPLICATION

\begin{tabular}{|l|l|l|}
\hline \multicolumn{1}{|c|}{ Feature } & \multicolumn{1}{|c|}{$\begin{array}{c}\text { KivaNS } \\
\text { application }\end{array}$} & \multicolumn{1}{|c|}{ EJS Applets } \\
\hline Setup difficulty & Middle-high & Very low \\
\hline Start time & Middle & Immediate \\
\hline Workspace & Muti-document & Specific form \\
\hline Learning difficulty & Middle & Very low \\
\hline $\begin{array}{l}\text { Can be embedded in web or } \\
\text { LMS }\end{array}$ & No & Yes \\
\hline Design of new networks & Yes & No \\
\hline Complex networks & Yes & No \\
\hline Kind of simulated networks & A wide range & Specific examples \\
\hline $\begin{array}{l}\text { Change of the simulation } \\
\text { parameters }\end{array}$ & $\begin{array}{l}\text { Requires a } \\
\text { reconfiguration of } \\
\text { the scheme }\end{array}$ & $\begin{array}{l}\text { A simulation can } \\
\text { be parameterized }\end{array}$ \\
\hline Main aim & $\begin{array}{l}\text { Network design } \\
\text { and simulation }\end{array}$ & $\begin{array}{l}\text { Show how a } \\
\text { network works }\end{array}$ \\
\hline $\begin{array}{l}\text { Multiplatform, free and } \\
\text { open source }\end{array}$ & Yes & Yes \\
\hline Requirements & Java JRE & $\begin{array}{l}\text { JRE and } \\
\text { Web-browser }\end{array}$ \\
\hline
\end{tabular}

\section{COURSE ORganization}

Nowadays, some LMS/CMS (Learning/Content Management System) are used to develop the educational strategy in Universities [4]. In this work, Moodle has been used to include activities and resources for Computer Networks subject (Fig. 8). Specifically, some SCORM packages have been exported to Moodle. The exported SCORM package has a runtime API and a data model used for communication between content objects and Moodle. In addition, the SCORM package provides a sequence to navigate during the learning activities. SCORM packages allow teachers to include documentation developed with different programs as hypertext web pages. They can even include dynamic information such as quizzes (Fig. 9), Java code and simulations (Figs. 5 and 6), Adobe flash presentations and other sources which can be executed in a modern web browser.

The student's assessment is a key item of the learning process for the university students. In the SCORM practice guide have been embedded some quizzes and exercises in order to permit the students to self-check whether they have learned and to measure their level acquired according to educative objectives required by teachers. Also, the students can revise those aspects whose comprehension is weaker because the quizzes for selfassessment have been designed to include an aid or 'how to'. An example of these SCORM guides is shown in Fig. 9. This guide can be acceded from the links available in the course page (Fig. 8).

This way, the student can measure the acquired knowledge level before the examination by the teacher. Thus, the student can decide whether his/her learning results are suitable and he/she is ready for the final exam. Moreover, the teacher can follow, on-line, the student's learning and adapt better the contents and their difficulty levels.

There are four assessments made by teacher, one for each practical lesson. And each student requires of 8 work-study hours by lesson. The assessments are realized

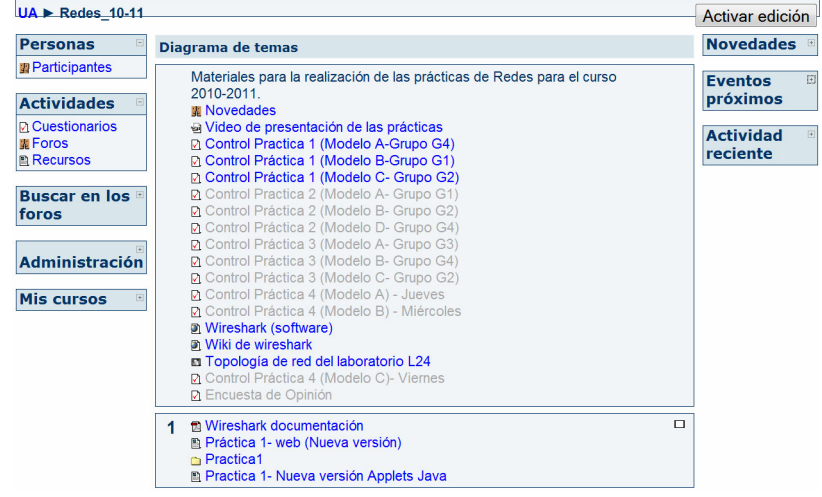

Figure 8. Computer Network subject in Moodle of University of Alicante (The course is taught in Spanish language)

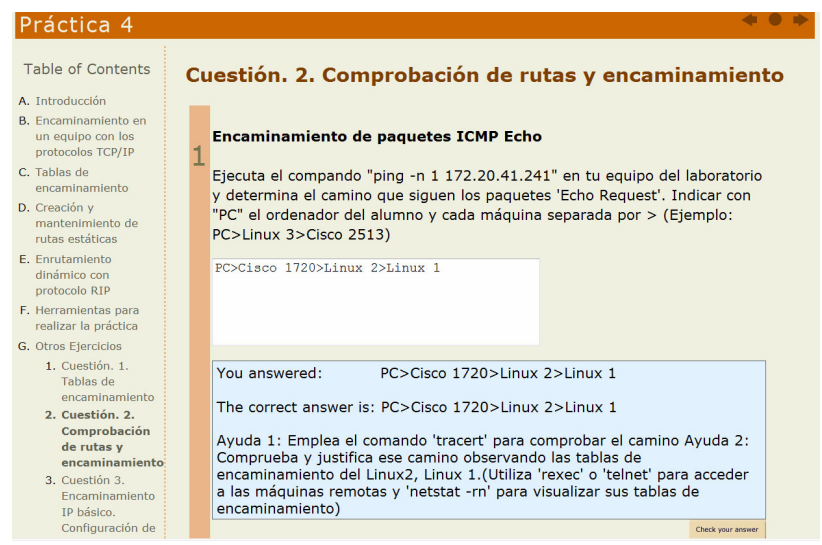

Figure 9. SCORM practice guide with questions for self-assesments of students in Computer Networks

Considerando la topología de red mostrada en la figura, en la que se muestra 1

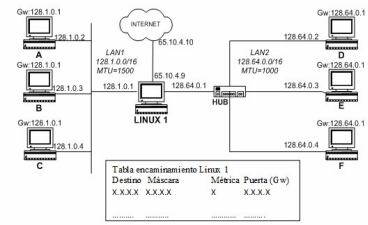

a) Se pide determinar las entradas directas que se mostrarían en la tabla de encaminamiento cuando se configuran los interfaces de red del dispositivo de interconexión LINUX1. Estas entradas servirán al LINUX para encaminar paquetes entre ambas redes.

a.1) ¿Qué entrada mostraría la tabla para el destino LAN2?

IP destino: 128.64.0.0 $\quad \checkmark$ Máscara: 0.0.0.0 $x \mathrm{IP}$

Gateway (puerta de enlace): 0.0 .0 .0

Figure 10. Example of question in Moodle (The course is taught in Spanish language)

from the quiz tool of Moodle. This tool allows teacher the automatic evaluation and correction. In Fig. 8, several links called "Control Practica N" lead to the final exams which are used to evaluate to the students. There are also different models of questionnaires for each exam and the questions have randomly chosen from database of questions. These questions have been grouped by similarity. Fig. 10 show an example of the question developed by quiz tool and answered by a student from Moodle. 


\section{LEARNING ASSESMENT}

In previous works, a survey has been arranged to determine the degree of satisfaction of the students in relation to the teaching methodology and the learning process with Moodle and SCORM packets [5] comparing with traditional teaching methodology, but without LCMS. These early versions of Computer Networks courses did not use Java applets, only text, static figures and interactive questions for self-assessment of the students in addition to a laboratory equipped with real networking devices. The main difference between the previous and the new version for the Computer Network course is that Java applets have been added to improve the constructive learning by means of simulating. Previously, only the real equipment had been used in the laboratory. Some years ago, the courses were only implemented with hypertext tools [11] and in recent years, the universities have been developing some simulation software $[7,12,13]$.

\section{A. Student's Opinion}

A survey has been arranged to determine the degree of satisfaction of the students in relation to the learning process when Java applets have been integrated with documentation in SCORM format. The survey consisted of 25 questions to evaluate different issues: the level of agree (Fig. 11), the acquired learning level (Fig. 12) with the implemented Java applets (Fig. 5) as well as the difficulty of learning and the level acquired knowledge, in general, using Java Applets together with real equipment. Moreover, the level of attendance to learn Computer Networks using the most common items of the learning process has been evaluated (Figs. 13 and 14).

In general, the great majority of students think that the required level to pass the course is appropiated. Not only, $93 \%$ of students have voted three on a scale between one (the lowest) and five (the highest) but also, they consider that the level of concepts and topics learned in the course have been high. The $76 \%$ of students think that the acquired level of knowledge is high and 19\% think that is very high.

Furthermore, with regard to the valuation of the features of applets, the student's opinion is very good. The level of disagree is low o very low, always below $10 \%$, as it can be observed in Fig. 11. On the contrary, at least the 55\% of students are satisfied with the support, usability, features, etc. which are offered by the Java applets.

\section{B. Assesment of the Approach's Efficency}

A study to analyze the acquired knowledge by the student such as the comprehension level about Link Layer of TCP/IP and its protocols Ethernet/ARP, the Internet Layer and its protocol IP, and device operations (hub, switch, bus, and router) is illustrated in Fig. 12. It shows how the learning level acquired by the students is high (63\%, 58\% and 47\% according to topics). Moreover, less than $1 \%$ of students think otherwise.

On the other hand, Figs. 13 and 14 show a comparison of student's opinon about what items of the course help to acquire a higher level of knowledge in the course of Computer Networks. This course consist of documentation about TCP/IP, teacher support to resolve questions and doubts, simulations with Java applets to observe operations with the protocols TPC/IP above mentioned and to analyze the real transmissions in the

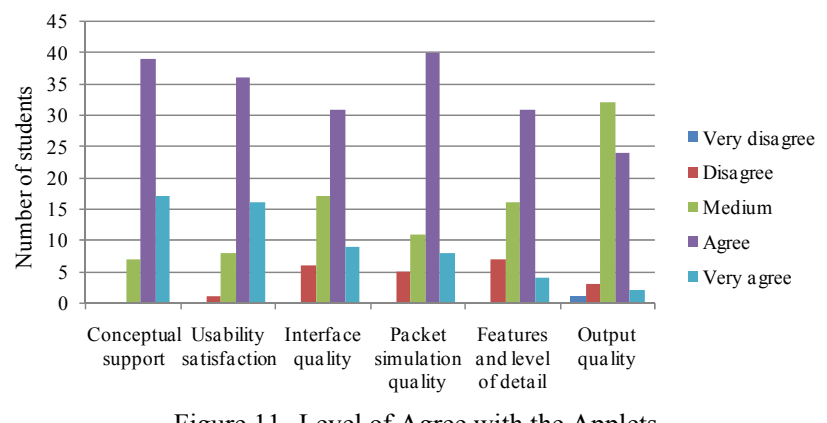

Figure 11. Level of Agree with the Applets

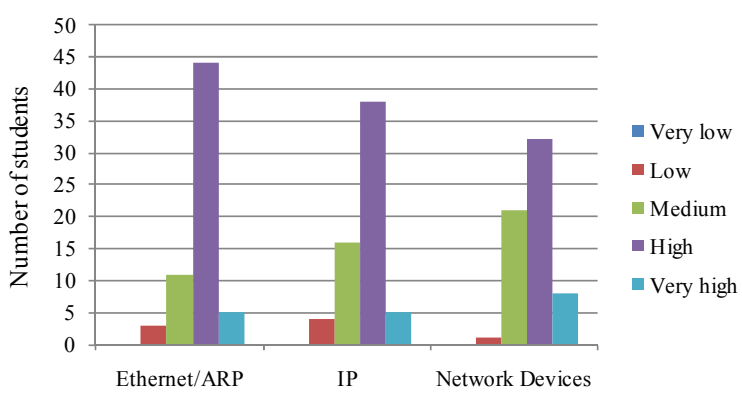

Figure 12. Acquired Knowledge Level with Applets

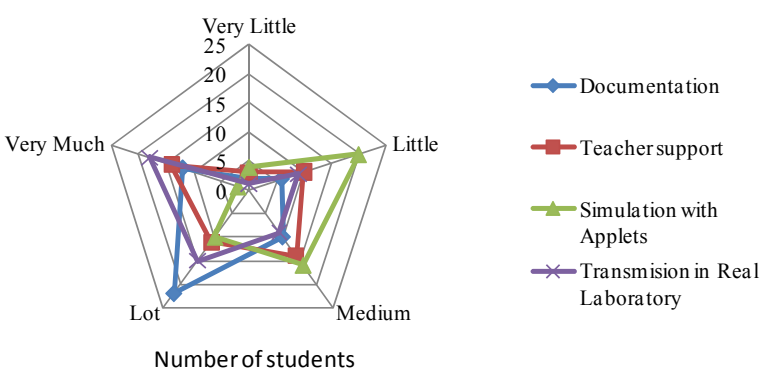

Figure 13. Student's opinion about attendance level to learn Computer Network concepts

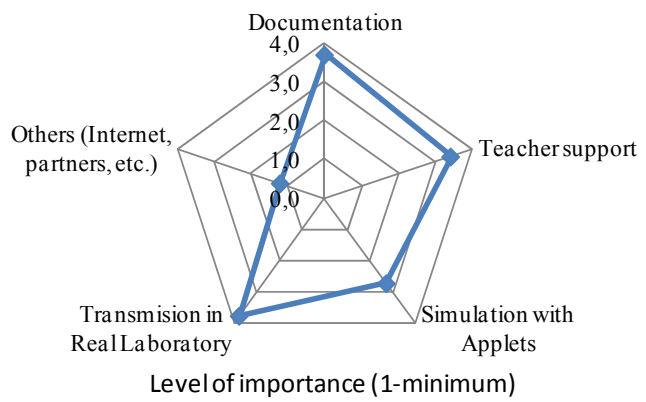

Figure 14. Comparison of average attendance level

laboratory using network devices and real equipment. The results show as the documentation and transmision in real laboratory have been the top rated (Fig. 14).

However, the simulation with Java applets obtain a half level importance 2.7 out of 5 againts the documentation and tranmision which are voted with 3.7 and 3.8 of 5 (Fig. 14). The simulation with Java applets is useful or very useful for the $54 \%$ of students although the $38 \%$ of them think that its usefulness is small if it is compared with a transmision with real network devices. Nevertheless, the students have commented that the applets offer advantages such as experience from home and clarify the 
understanding of general topics about the subject (routing, encapsulation, fragmentation, etc.). Therefore, they are an ideal complement for the real network and the experimentation. To conclude, according with the opinon extracted from surveys, it is possible that the simulation with applets have not had a higher value of acceptance comparing with the equipment in real laboratory because they have limitations of animation and reconfigurability.

\section{Grades acquiered}

The subject of Computer Networks is structured in 75 hours. Particularly, only 45 have been taught and evaluated with the help SCORM documents under LCMSMoodle in the three academic years: courses 2008, 2009 and 2010. In addition, in the last year, course 2010, 66 students have used Java applets integrated in SCORM documents to improve their learning. In the previous courses, years 2008 and 2009 have been evaluated 106 and 88 students, respectively. Nevertheless, these two previous years the SCORM documents did not have Java applets.

The Spanish Academic Grading System has been used to measure the reached level of knowledge. But, this system has been transformed to the most popular grading system, the USA grades which use discrete evaluation in the form of letter grades. To do this, an adaptation of Rackham School of Graduate Studies at the University of Michigan has been used in this paper. The Spanish Grade uses a scale between 0 and 10 and the USA Grade (US GPA) uses a scale between 0 and 4 . These grades can be assigned in letters as shows the Table II.

Analyzing the results of students during the past three academic courses can be deduced that the efficacy of learning is better. On the one hand, Fig. 15 shows how the students have obtained higher grades when they have used Java applets to learn concepts and topics about Link Layer. In general, there are more students with a success rate greater than $65 \%$, even greater than $90 \%$. At the same time, the students who do not pass the exam are significantly reduced in this last academic course. On the other hand, the results of the learning about Network Layer of TCP/IP are better too (Fig. 16). Similarly, in this last course, the number of students who do not pass the exam has decreased with respect to the previous years. Consequently, the number of students with a success rate over $65 \%$ has been increased using Java applets. However, the students with an excellent assessment result have only improved if are compared to 2008 but not with respect to 2009 .

\section{CONCLUSIONS}

This paper presents a practical approach for b-learning methodology. The work was done as part of the teaching method for Computer Network subject during the last course, in 2010. However, the general ideas presented here are applicable to other engineering or experimental science courses. The functionality of educational resources as digital documents, web packages with embedded interaction tools such as Java applets for simulations have been used to provide flexibility and autonomy to the learning process. The course can be followed without a face-to-face teacher support and from home. This course has been implemented in the platform
TABLE II.

EQUIVALENCE APPROXIMATED AMONG GRADES

\begin{tabular}{|c|c|c|c|}
\hline \multirow{2}{*}{$\begin{array}{l}\text { Percentage } \\
\text { s } \\
\text { Hits/Faults }\end{array}$} & \multicolumn{3}{|c|}{$\begin{array}{c}\text { Assesments/Qualifications in Educational } \\
\text { systems }\end{array}$} \\
\hline & $\begin{array}{c}\text { in } \\
\text { paper }\end{array}$ & Spain & USA \\
\hline $96-100 \%$ & $\mathrm{~A}+$ & Excellent (9.5-10] & A (3.8-4] \\
\hline $91-95 \%$ & A & Excellent [9-9.5] & A $(3.6,3.8]$ \\
\hline $86-90 \%$ & A- & Very Good (8.5-9) & B $(3.4,3.6]$ \\
\hline $81-85 \%$ & $\mathrm{~B}+$ & Very Good [8-8.5] & $\mathrm{B}(3.2,3.4]$ \\
\hline $76-80 \%$ & $\mathrm{~B}$ & Very Good $(7.5,8)$ & $\mathrm{C}(3,3.2]$ \\
\hline $70-75 \%$ & B- & Very Good $[7,7.5]$ & $\mathrm{C}(2.8,3]$ \\
\hline $67-69 \%$ & $\mathrm{C}+$ & Good (6.6.,7) & $\mathrm{D}(2.6,2.8]$ \\
\hline $63-66 \%$ & $\mathrm{C}$ & Good $(6.2,6.6]$ & $\mathrm{D}(2.5,2.6]$ \\
\hline $60-62 \%$ & C- & Good $[6,6.2]$ & $\mathrm{D}(2.4,2.5]$ \\
\hline $57-59 \%$ & $\mathrm{D}+$ & Sufficient $(5.6,6)$ & $\mathrm{E}(2.2,2.4]$ \\
\hline $53-56 \%$ & $\mathrm{D}$ & Sufficient $(5.2,5.6)$ & $\mathrm{E}(2.1,2.2]$ \\
\hline $50-52 \%$ & D- & Sufficient $[5,5.2]$ & $\mathrm{E}[2,2.1]$ \\
\hline$<50 \%$ & $\mathrm{E}, \mathrm{F}$ & Failure [0-5) & $\mathrm{F}[0-2)$ \\
\hline
\end{tabular}

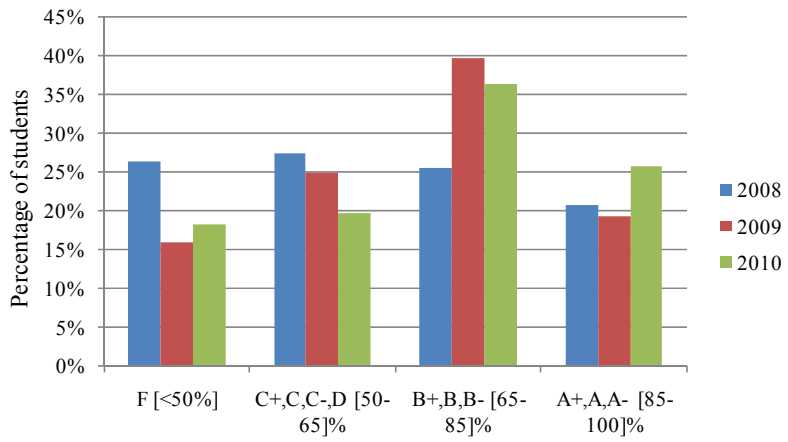

Figure 15. Grades acquired about Link Layer and Network Devices of TCP/IP

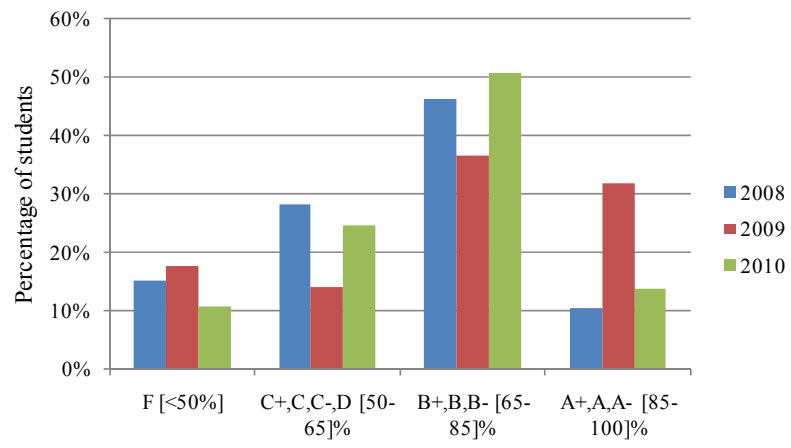

Figure 16. Grades acquired about Network Layer of TCP/IP

Moodle, which s accessible from the institutional LMS of the Alicante University. Over the last three years, the number of added educational resources has been increasing. Firstly, the digital documents and web pages, secondly the assessment tests and self-tests for training through the quiz module and now this last year, the interactive simulations without real network device by means of the integration of KivaNS in EJS. The application presented in this paper has been developed as an autonomous virtual laboratory to carry out experiments about TCP/IP protocols. Furthermore, these innovations introduced in the subject can mean important changes and they can influence to qualification mechanisms of topics 
and skills for the Computer Network course. For this reason, the authors have measured the satisfaction degree of the students when they use these new tools. The result is positive. Moreover, the progressive roll-out of these educational developments has permitted to teachers determine if the learning grades acquired are better than when other educational methodologies were used to teach Computer Networks.

\section{ACKNOWLEDGMENT}

This work is supported by the "Education Science Institute" and the "Technology \& Educational Innovation Vice-President Office" of the University of Alicante through the aid "Technologic \& Educative Research Groups (GITE)".

\section{REFERENCES}

[1] T. Clausen, "Undergraduate engineering education challenged by the Bologna declaration," IEEE Trans. on Education, vol. 48(2), pp. 213-215, May 2005. doi:10.1109/TE.2005.846045

[2] F.A. Candelas and P. Gil, "Practical experiments with KivaNS: a virtual laboratory for simulating IP routing in Computer Networks subjects," Research Reflections and Innovations in Integrating ICT in Education, vol 3, pp. 1415-1418, April 2009.

[3] N. Hoic-Bozic, V. Mornar, and I. Boticki, "A blended learning approach to course design and implementation," IEEE Trans. on Education, vol. 52 (1), pp. 19-30, 2009. doi:10.1109/ TE.2007.914945

[4] B. Chapman, LMS KnowledgeBase 2010: In-depth profiles of 90+learning management systems, with custom comparison across 200+features, Brandon Hall Research, 2009.

[5] P. Gil, F.A. Candelas, J. Pomares, S.T. Puente, J.A. Corrales, et al, "Using Moodle for an automatic individual evaluation of student's learning," 2nd Int. Conf. on Computer Supported Education, pp. 189-194, April 2010.

[6] C.A. Jara, F.A. Candelas, F. Torres, S. Dormido, F. Esquembre, et al, "Real time collaboration of virtual laboratories through the Internet," Computers \& Education, vol. 52(1), pp. 126-140, 2009. doi:10.1016/j.compedu.2008.07.007

[7] Java web site: http://www.java.com (April 2011)

[8] KivaNS web page: http://aurova.ua.es/kiva/indexi.html (April 2011)

[9] EJS web page: http://www.um.es/fem/EjsWiki/ (April 2011)
[10] A. Koohang, L. Riley, and T. Smith, "E-Learning and constructivism: from theory to application," Interdisciplinary J. of E-Learning and Learning Objects, vol. 5(1), pp. 91-109, January 2009.

[11] C. Chou, "Developing hypertext-based learning courseware for computer networks: the macro and micro stages," IEEE Trans.on Education, vol. 42(1), pp. 39-44, February 1999. doi:10.1109/ 13.746333

[12] M. Wannous and H. Nakano, "NVLab, a networking virtual webbased laboratory that implements virtualization and virtual network computing technologies," IEEE Tran. on Learning Technologies, vol. 3(2), pp. 129-138, April 2010. doi:10.1109/ TLT.2009.31

[13] P. Gil, F.A. Candelas and C.A. Jara, "Constructive Learning for Networks Courses based on Compact Simulations and SCORM" 2nd Int. Conf. IEEE Engineering Education, pp. 110-115, April 2011.

\section{AUTHORS}

P. Gil is with the Department of Physics, System Engineering and Signal Theory, University of Alicante, Carretera de San Vicente del Raspeig s/n, 03690 San Vicente del Raspeig, Alicante, Spain (e-mail: pablo.gil@ua.es).

F. A. Candelas is with the Department of Physics, System Engineering and Signal Theory, University of Alicante, Carretera de San Vicente del Raspeig s/n, 03690 San Vicente del Raspeig, Alicante, Spain (e-mail: francisco.candelas@ua.es).

C. A. Jara is with the Department of Physics, System Engineering and Signal Theory, University of Alicante, Carretera de San Vicente del Raspeig s/n, 03690 San Vicente del Raspeig, Alicante, Spain (e-mail: cajb@dfists.ua.es).

This work was supported in part by the Education Science Institute and the Technology \& Educational Innovation Vice-President Office of the University of Alicante through the aid "Technologic \& Educative Research Groups".

This article is an extended version of a paper presented at the IEEE EDUCON20211 Conference, held from April 4th-6th, 2011, in Amman, Jordan. Received April 14 ${ }^{\text {th }}$, 2011. Published as resubmitted by the authors May $2^{\text {nd }}, 2011$. 